Published in final edited form as:

Neuroimage. 2013 November 1; 81: 110-118. doi:10.1016/j.neuroimage.2013.05.030.

\title{
Real-time fMRI links subjective experience with brain activity during focused attention
}

\author{
Kathleen A. Garrison ${ }^{a}, \S$, Dustin Scheinost ${ }^{b}, \S$, Patrick D. Worhunsky ${ }^{a}$, Hani M. Elwafia ${ }^{a}$, \\ Thomas A. Thornhill IVa ${ }^{\text {, Evan Thompson }}{ }^{c}$, Clifford Saron ${ }^{d}$, Gaëlle Desbordes ${ }^{e}$, Hedy \\ Kober $^{\mathrm{a}}$, Michelle Hampson ${ }^{\mathrm{b}}$, Jeremy R. Gray ${ }^{\mathrm{a}, \mathrm{f}}, \mathbf{R}$. Todd Constable ${ }^{\mathrm{b}}$, Xenophon \\ Papademetris $^{\mathrm{b}}$, and Judson A. Brewer ${ }^{\mathrm{a},{ }^{*}}$
}

Kathleen A. Garrison: kathleen.garrison@yale.edu; Dustin Scheinost: dustin.scheinost@yale.edu; Patrick D. Worhunsky: patrick.worhunsky@yale.edu; Hani M. Elwafi: hani.elwafi@gmail.com; Thomas A. Thornhill: thomas.thornhill@yale.edu; Evan Thompson: evan.thompson@utoronto.ca; Clifford Saron: cdsaron@ucdavis.edu; Gaëlle Desbordes: gdesbord@nmr.mgh.harvard.edu; Hedy Kober: hedy.kober@yale.edu; Michelle Hampson: michelle.hampson@yale.edu; Jeremy R. Gray: jeremy.gray@yale.edu; R. Todd Constable: todd.constable@yale.edu; Xenophon Papademetris: xenophon.papademetris@yale.edu

aDepartment of Psychiatry, Yale University School of Medicine, 300 George St. Suite 901, New Haven CT, 06511

bDepartment of Diagnostic Radiology, Yale University School of Medicine, PO Box 208042, New Haven CT, 06511

'Department of Philosophy, University of Toronto, 170 St. George Street, Room 428, Toronto, Ontario, Canada M5S 1A1

${ }^{\mathrm{d} C e n t e r}$ for Mind and Brain, University of California Davis, 267 Cousteau Place, Davis, CA 95618

${ }^{e}$ Athinoula A. Martinos Center for Biomedical Imaging, Massachusetts General Hospital, 149 Thirteenth St \#2301, Boston, MA 02129

\section{Abstract}

Recent advances in brain imaging have improved the measure of neural processes related to perceptual, cognitive and affective functions, yet the relation between brain activity and subjective experience remains poorly characterized. In part, it is a challenge to obtain reliable accounts of participant's experience in such studies. Here we addressed this limitation by utilizing experienced meditators who are expert in introspection. We tested a novel method to link objective and subjective data, using real-time fMRI (rt-fMRI) to provide participants with feedback of their own brain activity during an ongoing task. We provided real-time feedback during a focused attention task from the posterior cingulate cortex, a hub of the default mode network shown to be activated during mind-wandering and deactivated during meditation. In a first experiment, both meditators and non-meditators reported significant correspondence between the feedback graph and their subjective experience of focused attention and mind-wandering. When instructed to volitionally decrease the feedback graph, meditators, but not non-meditators, showed significant deactivation

\footnotetext{
(c) 2013 Elsevier Inc. All rights reserved.

"To whom correspondence should be addressed: Judson A. Brewer, Department of Psychiatry, Yale University School of Medicine, 300 George St. Suite 901, New Haven, CT, 06511, Phone: 203-937-4840, Fax: 203-937-3478, judson.brewer@ yale.edu.

$\$$ Authors contributed equally to this work.

f Present address: Department of Psychology, Michigan State University, Psychology Building, East Lansing, MI 48824

Publisher's Disclaimer: This is a PDF file of an unedited manuscript that has been accepted for publication. As a service to our customers we are providing this early version of the manuscript. The manuscript will undergo copyediting, typesetting, and review of the resulting proof before it is published in its final citable form. Please note that during the production process errors may be discovered which could affect the content, and all legal disclaimers that apply to the journal pertain.

The authors declare no competing financial interests.
} 
of the posterior cingulate cortex. We were able to replicate these results in a separate group of meditators using a novel step-wise rt-fMRI discovery protocol in which participants were not provided with prior knowledge of the expected relationship between their experience and the feedback graph (i.e., focused attention versus mind-wandering). These findings support the feasibility of using rt-fMRI to link objective measures of brain activity with reports of ongoing subjective experience in cognitive neuroscience research, and demonstrate the generalization of expertise in introspective awareness to novel contexts.

\section{Keywords}

real-time neurofeedback; real-time fMRI; focused attention; posterior cingulate cortex; default mode network; meditation

\section{Introduction}

Recent advances in brain imaging have improved the measure of neural processes underlying human experience, yet the relation between objective measures of brain activity and subjective experience remains poorly characterized (Lutz and Thompson, 2003). Efforts to link objective and subjective data are challenged by inaccuracy or bias in self reports (Hurlburt and Heavey, 2001) and the potential to disrupt or modify an experience by generating a contemporaneous self report, among others (Schooler, 2002).

Recent strategies have improved the correspondence of objective and subjective data by probing subjects to report on their ongoing experience during standard neuroimaging. A recent study probed subjects during a sustained attention task during fMRI to ask whether their attention was focused and if they were on-task (Christoff et al., 2009). When they reported being off-task, subjects showed activity in the default mode network (DMN), a network implicated in mind-wandering and self-referential processing (Whitfield-Gabrieli et al., 2011). DMN activity was greatest when subjects were unaware of their own mindwandering, but made mistakes on the task. In another study (Hasenkamp et al., 2011), meditators were asked to practice focused attention on the breath meditation and press a button any time their mind wandered. Again, DMN activity was related to mind-wandering. These methods improve objectivity, yet still disrupt experience, and garner limited subjective information, that may be confounded by reverse inference of cognitive processes from brain activity (Christoff et al., 2009; Poldrack, 2006).

A novel way to overcome these limitations is to use real-time fMRI (rt-fMRI) to provide feedback of brain activity continuously during an ongoing task. Rt-fMRI combines the high spatial resolution of fMRI with improved temporal resolution lacking in blocked design fMRI, where subjects report averaged experience over the course of a block interval, rather than momentary experience. In so doing, rt-fMRI may allow for tighter coupling between real-time reports of experience and concomitant measures of brain activity. In theory, this method may be used to confirm group and time-averaged results at the individual subject level, in real time.

In this study, we link subjective experience with brain activity on a moment-to-moment basis during a focused attention task, by using rt-fMRI to provide feedback from the posterior cingulate cortex (PCC), a hub of the DMN shown to be activated during mindwandering and self-referential processing, among other activities such as remembering the past and predicting the future (Whitfield-Gabrieli et al., 2011) and deactivated during three types of meditation - focused attention on the breath, lovingkindness, and choiceless awareness (Brewer et al., 2011). We used the PCC for neurofeedback rather than another 
hub of the DMN (i.e., the mPFC) because PCC deactivation was the most robust finding associated with experienced meditators in our prior study (Brewer et al., 2011), and based on the purported role of the $\mathrm{mPFC}$ in relaying information to the PCC, rather than being directly involved in self-related processing (Ongur and Price, 2000). This study was designed to test the feasibility of using rt-fMRI to link ongoing subjective experience with an objective measure of brain activity in real time. We use an advantageous group, experienced meditators, who are practiced at both focused attention and monitoring moment-to-moment experience, and non-meditators as a control group.

\section{Materials and methods}

To assess whether real-time fMRI feedback can be used to more tightly couple an objective measure of brain activity with subjective experience, we conducted two studies. In Experiment 1A, experienced meditators and non-meditators were provided real-time feedback from the PCC or a control region during a focused attention task, and asked to rate how well the feedback graph corresponded with their subjective experience of focused attention or mind-wandering. In Experiment 1B, meditators and non-meditators were then instructed to volitionally decrease the feedback graph, in order to provide objective validation of their subjective experience. In Experiment 2, to replicate Experiment 1 without the potential confounds of experiential anchors for the graph -focused attention and mindwandering -provided in the earlier experiment, a separate group of meditators took part in a novel step-wise rt-fMRI blinded discovery protocol in which they were instructed to use their subjective experience during the task to discover the relationship between their experience and the feedback graph. All participants gave informed consent in accordance with the Yale University Human Investigation Committee.

\subsection{Experiment 1}

\subsubsection{Experiment 1A}

2.1.1.1. Participants: 22 meditators and 22 non-meditators took part in Experiment 1A. All participants were right-handed and case-control matched for gender, race, education, and employment status (Table 1). Meditators were drawn from the Theravada tradition. One meditator additionally practiced Buddhist non-dual meditation. Meditators reported a total of $9249 \pm 1449$ (mean \pm standard error of the mean) practice hours over $12.0 \pm 1.6$ years, consisting of both daily practice and retreats. Non-meditators reported no prior meditation experience.

2.1.1.2. fMRI task: Experiment $1 \mathrm{~A}$ included six 3.5 -minute runs. Each run consisted of a 30-second active baseline task designed to elicit self-referential processing, followed by a 3minute focused attention task. For the active baseline task, individuals viewed adjectives and mentally decided whether or not the words described them (Kelley et al., 2002). This task was used to provide a more standard baseline between groups than rest, given that we have previously found differences in DMN activity between meditators and non-meditators at rest (Brewer et al., 2011). For the focused attention task, traditional focused attention on the breath meditation instructions were operationalized for the fMRI setting (see Focused attention task instructions). From onset of the focused attention task, a column graph was back-projected onto a screen visible to participants in the scanner, and a new column was plotted every time to repetition ( $\mathrm{TR}=2$ seconds). Instructions were: "A graph will be displayed on the screen that will track the activity of a certain brain area involved in selfrelated processing. While you are meditating, the graph will have an upward, red signal if you engage in self-related processes such as when your mind wanders, and a downward, blue signal if you are fully absorbed in concentrating on the breath." Participants were instructed to practice focused attention on the breath, letting the graph rest in the background 
of their awareness, and to check in with the graph periodically to assess how it corresponded with their experience. They were told that due to signal processing, there would be a 4 to 8 second delay between their brain activity and the graph [based on the time course of the hemodynamic response function], and thus they should check the graph after noticeable periods of focused attention or mind-wandering, and then immediately return their attention to their breath. Their aim was to determine how well the graph corresponded with their momentary subjective experience. Participants were also instructed that they may receive feedback from different brain regions during separate runs, and to consider each run individually. Participants practiced the study procedure to proficiency outside the scanner, prior to scanning.

2.1.1.3. Focused attention task instructions: Standard mindfulness concentration meditation instructions were operationalized for fMRI, as follows: "During the meditation, please pay attention to the physical sensation of the breath wherever you feel it most strongly in the body. Follow the natural and spontaneous movement of the breath, not trying to change it in any way. Just pay attention to it. If you find that your attention has wandered to something else, gently but firmly bring it back to the physical sensation of the breath" (Brewer et al., 2011; Gunaratana, 2002).

2.1.1.4. Self report: After each run, participants were asked to respond verbally over the scanner intercom and: (1) rate how well the graph corresponded with their moment-tomoment subjective experience of focused attention on the breath, in blue, and mindwandering, in red, from $0=$ not at all to $10=$ perfectly; (2) briefly describe their rating i.e., to concisely describe what about the graph did or did not correspond with their subjective experience; and (3) rate how well they were able to follow the instructions from $0=$ not at all to $10=$ perfectly.

2.1.1.5. Real-time fMRI feedback: The feedback graph shown to participants during focused attention in each run showed percent change in the blood oxygenation leveldependent (BOLD) signal in a region of interest (see Region of interest definition) relative to baseline. For runs 1-4, the graph displayed feedback from the PCC. Runs 5-6 were randomized to display feedback from the PCC or a control region, the posterior parietal cortex. The PCC was used for feedback during the focused attention task because PCC activity has been shown to robustly increase during self-referential processing (Kelley et al., 2002; Mason et al., 2007; Northoff et al., 2006; Weissman et al., 2006), and, conversely, to decrease during different types of meditation (Brewer et al., 2011). The posterior parietal cortex was used as a control region because it is considered a node of the DMN that shows tight temporal coupling with the PCC, but activity in the posterior parietal cortex is not associated with self-referential processing (Andrews-Hanna et al., 2010; Northoff and Bermpohl, 2004; Northoff et al., 2006; Whitfield-Gabrieli et al., 2011). Thus, the posterior parietal cortex should show a comparable pattern of activity to the PCC, but should not correspond to subjective experience of focused attention. After run 6, participants were informed which of runs 5-6 showed feedback from the control region.

2.1.1.6. Image data acquisition: Participants were scanned using a Siemens 1.5 Tesla Sonata MRI with standard eight-channel head coil. A high-resolution anatomical scan was acquired using an MPRAGE sequence (TR/TE $=2530 / 3.34 \mathrm{~ms}, 160$ contiguous sagittal slices, slice thickness $1.2 \mathrm{~mm}$, matrix size $192 \times 192$, flip angle $=8^{\circ}$ ). A lower resolution T1weighted anatomical scan was then acquired: $\mathrm{TR} / \mathrm{TE}=500 / 11 \mathrm{~ms}$, field of view $=220 \mathrm{~mm}$, slice thickness $=4 \mathrm{~mm}$, gap $=1 \mathrm{~mm}, 25 \mathrm{AC}-\mathrm{PC}$ aligned axial-oblique slices. Functional image acquisition began at the same slice location as the T1 scan. Functional images were acquired using a T2*-weighted gradient-recalled single shot echoplanar sequence: $\mathrm{TR} / \mathrm{TE}=$ 
2000/35ms, flip angle $=90^{\circ}$, bandwidth $=1446 \mathrm{~Hz} /$ pixel, matrix size $=64 \times 64$, field of view $=220 \mathrm{~mm}$, voxel size $=3.5 \mathrm{~mm}$, interleaved, 210 volumes (after 2 volumes were acquired and automatically discarded). Ten additional functional volumes were acquired prior to realtime feedback, two were discarded, and the remaining eight volumes were used for registering single subject reference space for motion correction and region of interest definition.

2.1.1.7. Region of interest definition: Gray matter regions of interest (ROIs) for real-time feedback were defined using Bioimage Suite (www.bioimagesuite.org). For the PCC, we used reported activation coordinates in Brewer et al. (Brewer et al., 2011) for the local maximum for between-group differences (meditators versus non-meditators) during a focused attention on the breath meditation task $(-6,-60,18)$. For the posterior parietal cortex, we used anatomically defined coordinates $(-55,-51,19)$ in the intraparietal sulcus. 9 $\mathrm{mm}$ diameter spheres (volume $=461 \mathrm{~mm}^{3}$ ) were drawn from each of these coordinates. An additional gray matter mask was defined from all gray matter excluding the PCC and posterior parietal cortex $\left(960455 \mathrm{~mm}^{3}\right)$ on the MNI template brain.

2.1.1.8. Real-time image processing: Image processing for real-time feedback involved first transforming the ROIs from template space to single subject reference space using a series of linear and non-linear registrations, as described in prior studies (Hampson et al., 2011; Martuzzi et al., 2010). First, a non-linear transformation was used to warp the template brain to the individual 3D (MPRAGE) anatomical scan. Next, a linear registration of the individual 2D to 3D anatomical scan was performed. Finally, a linear registration of the short functional series to the 2D anatomical scan was performed. All transformations were estimated using the intensity-only component of the method implemented by Bioimage Suite, as reported previously (Papademetris et al., 2004), and were manually inspected for accuracy prior to feedback runs.

2.1.1.9. Real-time feedback display: The real-time feedback graph displayed to the participant was generated using a series of preprocessing steps for each frame of the fMRI time series, using the real-time fMRI feedback system described previously (Hampson et al., 2011; Scheinost et al., 2013). Briefly, each slice of a functional volume was reconstructed in real-time and each volume was motion corrected and analyzed immediately after acquisition. The mean activation of each ROI and the gray matter mask was calculated for each frame. To account for motion correction and partial volume effects near the edge of the brain, voxels with intensity less than $25 \%$ of the overall brain mean were excluded from mean activation. Any mean ROI activation with greater than $10 \%$ change from the previous frame was treated as an outlier and was replaced by the previous frame $\left(0^{\text {th }}\right.$ order interpolation). Mean ROI activation was temporally smoothed based on the last five values with a zero mean unit variance Gaussian kernel in order to provide less abrupt transitions in the feedback display from moment-to-moment. Percent signal change in the ROI during meditation was calculated relative to the ROI average value from the baseline task, and corrected for scanner drift by subtracting the percent signal change from the gray matter mask at each time point (deCharms et al., 2005). The corrected percent signal change relative to baseline was then presented as a graph to each participant in real-time using EPrime version 1.2 (pstnet.com). The entire processing stream from each functional volume acquisition to feedback display required less than 1-second delay. Corrected percent signal change was stored for offline analysis.

2.1.1.10. Statistical analysis: Statistical analyses used SPSS (ibm.com). In order to evaluate whether real-time feedback from the PCC corresponded to the subjective experience of focused attention and mind-wandering, in meditators and non-meditators, we calculated the 
mean correspondence rating for the self report question "How well did your experience correlate with the graph?" $(0=$ not at all, $10=$ perfectly $)$, for runs $1-4$, and performed between-group comparisons using an independent t-test. In order to evaluate whether participants could discriminate between feedback from the PCC and control region, the posterior parietal cortex, we compared mean correspondence ratings between ROIs from runs 5-6 using a paired t-test. We report mean \pm standard error of the mean.

\subsubsection{Experiment 1B}

2.1.2.1. Participants: 9 meditators and 11 non-meditators from Experiment $1 \mathrm{~A}$ took part in Experiment 1B (Table 1). This subset of meditators reported a total of $8803 \pm 3282$ practice hours over $9.5 \pm 2.4$ years, consisting of both daily practice and retreats. Experiment $1 \mathrm{~B}$ was conducted in an additional $7^{\text {th }}$ run added to Experiment 1A part-way through data collection; all subsequent participants completed this $7^{\text {th }}$ run.

2.1.2.2. Real-time feedback task: Experiment $1 \mathrm{~B}$ was comprised of the same 30-second active baseline task as in Experiment 1A, followed by an additional real-time feedback task (Figure 1). For the real-time feedback task, participants were given the following instructions: "Now you have gotten a chance to see how activation of this brain region correlates with meditation. Given what you have learned from the previous runs, in the next run, please see how much you can actively make the graph go blue." During the run, participants were again shown a graph of real-time feedback from the PCC as in Experiment 1A. Image acquisition, ROI definition, real-time image processing and feedback display were the same as in Experiment 1A.

2.1.2.3. Statistical analysis: Mean percent signal change in the PCC during real-time feedback was calculated and compared between groups using independent t-tests. We also tested for Pearson's correlations between percent signal change in the PCC and meditation experience (total lifetime hours).

2.1.2.4. Offline motion analysis: In order to ensure that feedback from the PCC was not due to subject motion during the real-time feedback task, we ran a correlation analysis between offline motion parameter estimates and percent signal change in the PCC during real-time feedback. Functional images were realigned for motion correction using SPM 8 (www.fil.ion.ucl.ac.uk/spm). Each resultant motion parameter was averaged across all slices for the run. A two-tailed correlation was tested between mean percent signal change in the PCC and each average motion parameter for each group (meditators/non-meditators), given that there may be group differences in movement during scanning. No significant correlation was found (controls: $\mathrm{x}: \mathrm{r}=.04, p=.91 ; \mathrm{y}: \mathrm{r}=-.25, p=.45 ; \mathrm{z}=.04 p=.9 ;$ pitch: $\mathrm{r}=.03, p$ $=.93 ;$ roll: $\mathrm{r}=.37, p=.26$; yaw: $\mathrm{r}=.21, p=.53$; meditators: $\mathrm{x}: \mathrm{r}=.25, p=.48 ; \mathrm{y}: \mathrm{r}=-.24, p$ $=.51 ; \mathrm{z}: \mathrm{r}=-.29, p=.41 ;$ pitch: $\mathrm{r}=-.11, p=.76 ;$ roll: $\mathrm{r}=-.25, p=.49$; yaw: $\mathrm{r}=-.2, p=$. 58). Additionally, no correlation was found between motion parameters and mean percent signal change in the PCC if the absolute value of the motion parameters were averaged. For simplicity, we present only the purely averaged parameters.

\subsection{Experiment 2}

2.2.1. Participants -10 meditators ( 9 right-handed, 1 ambidextrous) took part in Experiment 2 (Table 1). Meditators were drawn from a number of contemplative traditions including Theravada ( $\mathrm{N}=4)$, Zen ( $\mathrm{N}=3$ ), Catholic Contemplative $(\mathrm{N}=1)$, Catholic Contemplative and Zen ( $\mathrm{N}=1)$, and Gelug-pa of Tibetan Buddhism $(\mathrm{N}=1)$. Meditators reported a total of $10567 \pm 4276$ practice hours over $18.4 \pm 4.9$ years, consisting of both daily practice and retreats. 
2.2.2. Blinded discovery fMRI protocol-A subsequent experiment was then designed to replicate Experiment 1A without providing participants with experiential anchors for the feedback graph - such as focused attention in blue and mind-wandering in red - thus eliminating potential bias in how participants link their subjective experience to the feedback graph. The protocol was designed to allow participants to discover how the feedback graph corresponded to their own subjective experience of focused attention in realtime. This blinded discovery protocol was comprised of a 4-step series of fMRI runs progressing from (1) focused attention on the breath with offline feedback (feedback graph shown offline immediately after each run), (2) focused attention on a simulated graph with offline feedback, (3) focused attention with real-time feedback from the PCC, to (4) volitional decrease of the feedback graph. This stepwise protocol was designed to progress from the most naturalistic setting for focused attention (step 1), to focused attention using a dynamic graph as the object of focus (step 2), to focused attention with a graph of feedback from one's own brain activity in real-time (step 3), to volitional decrease of the feedback graph (step 4) (Figure 2). Step 1 included 4 runs because it was the most naturalistic setting for focused attention on the breath, and was thus crucial for discovering how one's own experience corresponded with the feedback graph, shown to participants offline. Steps 2-4 each included 3 runs. Each run was comprised of the same 30-second active baseline task as in Experiment 1, followed by the same focused attention meditation task, with the exception that the focused attention task was shortened from 3 minutes to 1 minute to aid recall of moment-to-moment experience. Pilot testing of the focused attention task using durations ranging from 30 seconds to 3 minutes, with meditators who were not included in the current analysis, yielded that 60 seconds was optimal for practicing focused attention and also recalling one's momentary subjective experience. Participants practiced the study procedure to proficiency outside of the scanner prior to scanning. Image acquisition, ROI definition, real-time image processing and feedback display were the same as in Experiment 1.

2.2.3. Blinded discovery fMRI protocol instructions-Participants were instructed that any time they viewed a graph of their brain activity, the graph would show activity from the same brain region, and their aim was to see whether or not activity in this brain region corresponded with their subjective experience. They were not provided experiential anchors for the feedback graph (e.g., focused attention/mind-wandering). Instead, they were instructed that increases in the graph, in red, indicated increased activity in the brain region, and decreases in the graph, in blue, indicated decreased activity in the same brain region. Specific instructions for each step were as follows:

2.2.3.1. Focused attention with offline feedback: For the first meditation task, after the word task, the screen will go blank. This will be your cue to meditate for about 60 seconds. During the meditation, please pay attention to the physical sensation of the breath wherever you feel it most strongly in the body. Follow the natural and spontaneous movement of the breath, not trying to change it in any way. Just pay attention to it. If you find that your attention has wandered to something else, gently but firmly bring it back to the physical sensation of the breath. Please keep your eyes open.

2.2.3.2. Focused attention on a graph with offline feedback: For the second meditation task, after the word task, you will see a graph start to form, that will fill in a new line every 2 seconds. This is an arbitrary graph, and does not show your brain activity. We ask that when you see the graph start to form, you again meditate for 60 seconds, here using the graph as your object of meditation- just paying attention to the graph as you would any other object of focus or concentration such as your breath. Pay attention to the graph, not trying to change it in any way. If you find that your attention has wandered to something else, gently but firmly bring it back to the graph. Please keep your eyes open. 
2.2.3.3. Focused attention with real-time feedback: For the third meditation task, after the word task, you will see a similar graph start to form, and again we ask that you meditate for 60 seconds, using the graph as your object of meditation. Now the graph you see during the run will show relative activity in a particular region of your brain. Thus for these runs, the graph you see during the run may correspond with your experience. There is a 2-4 second delay between your brain activity and the graph, thus if the graph does correspond with your experience, it will do so with a delay of 2-4 seconds [Authors note: from Experiment 1B and pilot testing, meditators reported a shorter lag between their experience and the feedback graph than 4-8 seconds. This may be due to a differential hemodynamic response function across the brain (Miezin et al., 2000), with the PCC possibly having a shorter lag. We updated the instructions accordingly for Experiment 2]. It may be helpful to look back at short stretches of time to notice your experience in relation to how the graph changes. We ask that you meditate, using the graph as your object of meditation, and now also notice your moment-to-moment experience in relation to how the graph changes.

2.2.3.4. Volitional decrease of the feedback graph: For the final task, after the word task, you will see a similar graph start to form that will show relative activity in a particular region of your brain, and may correspond with your experience. For these runs, we will ask you to use your mind to make the graph go blue. You may draw from your experience over the previous runs. You will have 60 seconds.

2.2.4. Online feedback and self report-For steps $1-2$, after each run, participants were first asked to briefly describe their experience during the focused attention meditation. They were then shown a graph of their brain activity from the focused attention meditation period (offline feedback), and asked "How well did the graph correspond with your experience during the meditation, from $0=$ not at all to $10=$ perfectly". They were instructed to consider how the graph did or did not correspond with their experience during the focused attention task, for example, with their general experience of meditation, including mental effort, concentration, or mental state, and at the beginning, middle, or end of the focused attention task. For step 3, participants were again asked to briefly describe their experience during the focused attention meditation, and then to rate how well the graph they saw during the run (real-time feedback) corresponded with their experience. For step 4, participants were asked to briefly describe their experience during the focused attention meditation, to rate how well the graph they saw during the run corresponded with their experience, and additionally, to report what strategy they used to try to decrease the feedback graph.

2.2.5. Statistical analysis-Mean correspondence rating for the self-report question "How well did the graph correspond with your experience during the meditation from $0=$ not at all, $10=$ perfectly?" was calculated from step 3, focused attention with real-time feedback (3 runs). Mean percent signal change during real-time feedback was calculated for the PCC from step 4, volitional manipulation of the feedback graph (3 runs). One participant was unable to take part in step 4 due to arriving late, thus mean percent signal change during real-time feedback is calculated from $\mathrm{N}=9$. We used two-sided one-sample t-tests relative to zero to test for significance.

\section{Results}

\subsection{Experiment 1A}

In Experiment 1A, all participants reported success in following the instructions. Mean rating for runs 1-6 when participants were asked How well were you able to follow the 
instructions from $0=$ not at all to $10=$ perfectly was $8.8 \pm 0.1$ and did not differ between groups $\left(\mathrm{t}_{(42)}=.31, p=.78\right)$.

Meditators and non-meditators reported a significant correspondence between their subjective experience and the feedback graph for runs 1-4, such that focused attention corresponded with decreases in the graph, in blue, and mind-wandering and/or selfreferential processing corresponded with increases in the graph, in red. Mean correspondence rating for all participants between subjective experience and the feedback graph was $7.6 \pm 0.2$ and did not differ between groups $\left(\mathrm{t}_{(42)}=1.1, p=.29\right)$. Feedback graphs representing mean percent signal change from the PCC in run 1 and run 4 for an example meditator and non-meditator are displayed in Figure 3.

Participants were able to discriminate between real-time feedback from the PCC and from the posterior parietal cortex control region. For runs 5-6 when participants received feedback from the PCC and control region in a randomized order, mean correspondence rating for all participants was significantly higher for runs with feedback from the PCC (8.1 $\pm 0.3)$ as compared to runs with feedback from the posterior parietal cortex $\left(6.9 \pm 0.4 ; \mathrm{t}_{(42)}=\right.$ $3.1, p=.004)$. We note that the moderate mean correspondence rating found between subjective experience and feedback from the posterior parietal cortex was likely due to tight temporal coupling of the posterior parietal cortex with the PCC during focused attention, shown in prior studies in both meditators and non-meditators (Andrews-Hanna et al., 2010; Brewer et al., 2011).

\subsection{Experiment 1B}

For run 7 in which participants were asked to volitionally decrease the feedback graph, meditators showed significant PCC deactivation $(-0.26 \pm 0.1)$, as compared to nonmeditators $\left(0.07 \pm 0.09 \% ; \mathrm{t}_{(18)}=-2.40, \mathrm{p}=.028\right.$, Figure 4$)$. For meditators, no correlation was found between meditation hours and percent signal change in the PCC $(\mathrm{r}=-.08, p=$. $83)$.

\subsection{Experiment 2}

The aim of Experiment 2 was to replicate Experiment 1 without potential bias from experiential anchors for the real-time feedback graph, using a 4-step blinded discovery fMRI protocol, whereby participants discovered how their subjective experience corresponded with the feedback graph. We again assessed mean correspondence rating and percent signal change in the PCC. All participants reported coupling between their subjective experience of focused attention and decreases in the feedback graph representing PCC activity by the end of discovery step 1. Meditators again reported a significant correspondence between the feedback graph and their subjective experience during focused attention, as in Experiment 1A. From step 3, focused attention with real-time feedback, mean correspondence rating was $7.6 \pm 0.3\left(\mathrm{t}_{(9)}=25.4, p<.0001\right)$. Meditators again showed significant volitional decrease of the feedback graph, as in Experiment 1B. From step 4, volitional manipulation of the graph, mean percent signal change in the PCC was $-0.26 \pm 0.1 \%\left(\mathrm{t}_{(8)}=-2.71, p=.026\right.$; Figure 5). All participants reported using focused attention on the breath as their strategy for volitional decrease of the feedback graph.

\section{Discussion}

In this study, we used real-time fMRI neurofeedback to assess the coupling between a real time objective measure of brain activity in the PCC, and momentary subjective experience during a focused attention task, in meditators and non-meditators. In so doing, we 
demonstrated the feasibility of using rt-fMRI to link subjective mind states and objective measures of brain activity.

In Experiment 1A, we showed that a feedback graph representing PCC activity provided information closely related to ongoing subjective experience during a focused attention task in meditators and non-meditators. Both groups reported significant correspondence between their mental experience and the feedback graph during the task. Participants were further able to distinguish feedback from the PCC from feedback from a control brain region, reducing the likelihood of confirmation bias. A limitation of Experiment $1 \mathrm{~A}$ was the provision of experiential anchors for the feedback graph: participants were informed that decreases in the graph, in blue, represented focused attention, and increases in the graph, in red, represented mind-wandering. Such anchors for feedback are helpful for testing specific hypotheses by limiting the range of subjective experience to which participants pay attention, but also may bias participants' selfreport. To mitigate this potential confound, we replicated the study in Experiment 2, in which a separate group of meditators were able to learn how their own subjective momentary experience related to the feedback graph, without the use of experiential anchors for feedback. Participants had to, first, discover (in step 1, focused attention on the breath with offline feedback), and then evaluate (in step 3, focused attention with real-time feedback), how the feedback graph corresponded to their own subjective experience. Again meditators reported a significant correspondence between their experience and the feedback graph.

In both Experiments 1B and 2, we found that meditators were able to volitionally decrease the feedback graph, as assessed by PCC deactivation. Volitional decrease of the feedback graph may be considered, in part, a confirmation of correspondence ratings between subjective experience and the feedback graph. Because participants reported high correspondence between their subjective experience and the feedback graph, they should be able to use what they have discovered about how their experience relates to the feedback graph, to manipulate the graph. Meditators indeed showed significant PCC deactivation when asked to volitionally decrease the feedback graph. In contrast, non-meditators did not show PCC deactivation when asked to volitionally decrease the feedback graph. Meditators performance may have been due to their expertise at focused attention and monitoring of experience. However, interpretation of our results is limited beyond focused attention meditation in the research setting. Traditional meditation practices usually involve contextual components such as background beliefs, intentions for practice, and community support, among others; in the current study, focused attention was decontextualized (performed in an fMRI scanner), and these other factors were not measured. However, since meditators were long-term practitioners with significant life commitments to meditation practice, we cannot rule out that larger meaning components of the practice or memory of other practice contexts were active even during the decontextualized focused attention task. Due to these empirical differences, further studies are necessary to interpret our findings within the broader field of contemplative research, including in relation to cognitive and behavioral outcomes of contemplative practice.

\subsection{Utility of rt-fMRI feedback for cognitive neuroscience}

This set of novel findings supports the use of real-time fMRI neurofeedback in cognitive neuroscience research to assess the coupling between real-time feedback from brain activity and concurrent self-reports of mental states. To our knowledge, our study demonstrates the first use of rt-fMRI to link first-person experience with neuroimaging data in real time.

Prior studies have used blocked design fMRI with self-report to assess brain activity during various aspects of a cognitive process. For example, Mason et al. measured DMN activity during blocks of a novel or learned working memory task, in order to test whether training 
participants 'to boredom' on a task would lead to greater DMN activity associated with mind-wandering (Mason et al., 2007). As predicted, they found greater DMN activity and greater self-reported mind-wandering during learned than novel blocks, despite the identical task. Here we report that activity in a hub of the DMN, the PCC, is related to focused attention and mind-wandering in real time, and not just when averaged over an fMRI block. Our findings add convergent validity and improved temporal resolution to prior studies (Pagnoni, 2012). More broadly, our findings provide proof of concept that rt-fMRI may be used to more closely link activity in a particular brain region with varied aspects of a cognitive process. In addition, by having participants report their direct experience as it corresponds with feedback, the potential problem of reverse inference (Poldrack, 2006) is obviated. This does not imply that single brain regions such as the PCC can wholly account for complex cognitive processes such as focused attention or meditation, but instead, that targeted feedback may help to elucidate a set of processes related to networks of brain regions, such as those related to the DMN. To overcome this limitation, future studies may use real-time feedback from multiple hubs, or functional connectivity between hubs, to further refine our understanding of neural networks underlying such cognitive processes.

Prior work to link subjective experience with objective neuroimaging data has been limited in part by individual differences in subjective experience of mind states. For example, for meditation, the depth and quality of the meditative state may vary between individuals, or for an individual between meditation blocks, or even from moment-to-moment within a given meditation block. These inter- and intra-individual differences in experience contribute to the challenge of accurately characterizing underlying brain processes. Our experimental design takes advantage of this variability by asking participants to pay attention to how their own moment-to-moment experience changes, and to report how changes in their own experience relate to changes in the feedback graph. In this way, variability within individuals, and even variability within task blocks, does not confound results, but instead is utilized to more tightly couple subjective experience with brain activity. Directed methodologies such as this may also improve accuracy in more directly linking subjective experience to brain activity during a task and therefore increasing statistical power.

Our study has several additional limitations related to variability. First, a number of cognitive processes involved in the focused attention task may vary across individuals, including motivation, relaxation, interest, and so forth. Although correspondence between subjective experience and the feedback graph was significant, our findings are reduced to a single experiential dimension and we are unable to differentiate between focused attention and these other cognitive processes. For example, sub-regions of the PCC have demonstrated dissociable functional connectivity patterns above and beyond generalized PCC deactivation during a working memory task (Leech et al., 2011). To improve specificity, we provided feedback from a sub-region of the PCC robustly associated with self-referential processing, though again our paradigm was not designed to differentiate between specific elements therein (e.g. mindwandering vs. future thinking) (WhitfieldGabrieli et al., 2011). Further, we show that meditators are able to volitionally decrease the feedback graph with and without experiential anchors for the graph, and no meditator reported using a cognitive strategy other than related to focused attention to manipulate the graph. A further refinement would be to use multiple or extended runs, especially given the complexity of our task, which required both ongoing focused attention and evaluation of how one's experience corresponded to the graph. Successive runs might allow a more refined detection of which specific features of subjective experience relate to different aspects of the graph, such as highly associative thought, distraction and so on. In this way, task repetition would reduce variability. A previous study used a related approach to reduce noise resulting from varied cognitive strategy during a perceptual illusion task with 
electroencephalogram (Lutz et al., 2002). Self-reports were analyzed for recurrent patterns of cognitive strategy during the task, and runs were clustered and analyzed for brain activity related to each strategy. A similar design could be used with rt-fMRI by asking participants to notice which strategies they are using during an ongoing task in real time, and over successive or extended runs, to look for stable phenomenal invariants in how their task strategy relates to the feedback graph. This approach would further refine our understanding of how activity in a particular brain region relates to specific cognitive processes.

\subsection{Conclusions}

In sum, as demonstrated by this study, rt-fMRI provides a novel methodology for more tightly coupling the dynamics of subjective experience and changes in ongoing brain activity. Additional future uses include neurophenomenological investigations (Varela, 1996) in which specific subjective cognitive components of experience are linked to brain activity, and testing the robustness of cognitive strategies and tasks in real time (e.g. how reliably and to what degree does a new task actually activate or deactivate a hypothesized brain region in single subjects), among others. We also propose that experienced meditators provide a population of choice for future studies given their higher degree of introspective awareness and capacity to detect subtle changes in their subjective experience in real time.

\section{Acknowledgments}

This work was supported by the National Institutes of Health, National Institute on Drug Abuse (K12-DA00167 and 1R03DA029163-01A1 to JB), the U.S. Veterans Affairs New England Mental Illness Research, Education, and Clinical Center, and by private donations from Jeffrey C. Walker, Austin Hearst, and the 1440 Foundation. The funding sources had no role in conduct of the research or preparation of the article. We thank our participants for their time and effort; Joseph Goldstein and Ginny Morgan for input on meditation instructions; Jithendra Bhawnani, and Maolin Qiu for technical support with real-time fMRI; Alice Brewer, Matthew Sacchet and Reza Farajian for feedback on the manuscript; Marc Potenza, Kathleen Carroll, and the staff of the Yale Therapeutic Neuroscience Clinic, Hedy Sarofin and staff of the Yale Magnetic Resonance Research Center for their contributions.

\section{References}

Andrews-Hanna JR, Reidler JS, Sepulcre J, Poulin R, Buckner RL. Functional-Anatomic Fractionation of the Brain's Default Network. Neuron. 2010; 65:550-562. [PubMed: 20188659]

Brewer JA, Worhunsky PD, Gray JR, Tang YY, Weber J, Kober H. Meditation experience is associated with differences in default mode network activity and connectivity. Proceedings of the National Academy of Sciences of the United States of America. 2011; 108:20254-20259. [PubMed: 22114193]

Christoff K, Gordon AM, Smallwood J, Smith R, Schooler JW. Experience sampling during fMRI reveals default network and executive system contributions to mind wandering. Proceedings of the National Academy of Sciences of the United States of America. 2009; 106:8719-8724. [PubMed: 19433790]

deCharms RC, Maeda F, Glover GH, Ludlow D, Pauly JM, Soneji D, Gabrieli JD, Mackey SC. Control over brain activation and pain learned by using real-time functional MRI. Proceedings of the National Academy of Sciences of the United States of America. 2005; 102:18626-18631. [PubMed: 16352728]

Gunaratana, H. Mindfulness in Plain English. Somerville, MA: Wisdom Publications; 2002.

Hampson M, Scheinost D, Qiu M, Bhawnani J, Lacadie CM, Leckman JF, Constable RT, Papademetris X. Biofeedback of Real-Time Functional Magnetic Resonance Imaging Data from the Supplementary Motor Area Reduces Functional Connectivity to Subcortical Regions. Brain Connectivity. 2011; 1:91-98. [PubMed: 22432958]

Hasenkamp W, Wilson-Mendenhall CD, Duncan E, Barsalou LW. Mind wandering and attention during focused meditation: A fine-grained temporal analysis of fluctuating cognitive states. Neuroimage. 2011 
Hurlburt RT, Heavey CL. Telling what we know: describing inner experience. Trends in Cognitive Sciences. 2001; 5:400-403. [PubMed: 11520705]

Kelley WM, Macrae CN, Wyland CL, Caglar S, Inati S, Heatherton TF. Finding the self? An eventrelated fMRI study. Journal of Cognitive Neuroscience. 2002; 14:785-794. [PubMed: 12167262]

Leech R, Kamourieh S, Beckmann CF, Sharp DJ. Fractionating the default mode network: distinct contributions of the ventral and dorsal posterior cingulate cortex to cognitive control. The Journal of neuroscience : the official journal of the Society for Neuroscience. 2011; 31:3217-3224. [PubMed: 21368033]

Lutz A, Lachaux J-P, Martinerie J, Varela FJ. Guiding the study of brain dynamics by using firstperson data: Synchrony patterns correlate with ongoing conscious states during a simple visual task. Proceedings of the National Academy of Sciences. 2002; 99:1586-1591.

Lutz A, Thompson E. Neurophenomenology integrating subjective experience and brain dynamics in the neuroscience of consciousness. Journal of Consciousness Studies. 2003; 10(9):31-52.

Martuzzi R, Ramani R, Qiu M, Rajeevan N, Constable RT. Functional connectivity and alterations in baseline brain state in humans. Neuroimage. 2010; 49:823-834. [PubMed: 19631277]

Mason MF, Norton MI, Van Horn JD, Wegner DM, Graffon ST, Macrae CN. Wandering Minds: The Default Network and Stimulus-independent Thought. Science. 2007; 315:393. [PubMed: 17234951]

Miezin FM, Maccotta L, Ollinger JM, Petersen SE, Buckner RL. Characterizing the hemodynamic response: effects of presentation rate, sampling procedure, and the possibility of ordering brain activity based on relative timing. Neuroimage. 2000; 11:735-759. [PubMed: 10860799]

Northoff G, Bermpohl F. Cortical midline structures and the self. Trends in Cognitive Sciences. 2004; 8:102. [PubMed: 15301749]

Northoff G, Heinzel A, de Greck M, Bermpohl F, Dobrowolny H, Panksepp J. Selfreferential processing in our brain--A meta-analysis of imaging studies on the self. Neuroimage. 2006; 31:440-457. [PubMed: 16466680]

Ongur D, Price JL. The Organization of Networks within the Orbital and Medial Prefrontal Cortex of Rats, Monkeys and Humans. Cerebral Cortex. 2000; 10:206-219. [PubMed: 10731217]

Pagnoni G. Dynamical Properties of BOLD Activity from the Ventral Posteromedial Cortex Associated with Meditation and Attentional Skills. Journal of Neuroscience. 2012; 32:5242-5249. [PubMed: 22496570]

Papademetris, X.; Jackowski, A.; Schultz, R.; Staib, L.; Duncan, J. Integrated Intensity and PointFeature Nonrigid Registration. In: Barillot, C.; Haynor, D.; Hellier, P., editors. Medical Image Computing and Computer-Assisted Intervention. Berlin / Heidelberg: Springer; 2004. p. 763-770.

Poldrack RA. Can cognitive processes be inferred from neuroimaging data? Trends in Cognitive Sciences. 2006; 10:59-63. [PubMed: 16406760]

Scheinost D, Hampson M, Qiu M, Bhawnani J, Constable RT, Papademetris X. A Graphics Processing Unit Accelerated Motion Correction Algorithm and Modular System for Real-time fMRI. Neuroinformatics. 2013

Schooler JW. Re-representing consciousness: dissociations between experience and metaconsciousness. Trends Cogn Sci. 2002; 6:339-344. [PubMed: 12140084]

Varela FJ. Neurophenomenology: a methodological remedy for the hard problem. Journal of Consciousness Studies. 1996; 3:330-349.

Weissman DH, Roberts KC, Visscher KM, Woldorff MG. The neural bases of momentary lapses in attention. Nature Neuroscience. 2006; 9:971-978.

Whitfield-Gabrieli S, Moran JM, Nieto-Castan A, Triantafyllou C, Saxe R, Gabrieli JDE. Associations and dissociations between default and self-reference networks in the human brain. Neuroimage. 2011; 55:225-232. [PubMed: 21111832] 


\section{Highlights}

- Real-time fMRI feedback from the PCC corresponds to subjective focused attention

- Feedback corresponded to experience in both meditators and non-meditators

- Meditators deactivated the PCC upon volitional decrease of the feedback graph

- Results were replicated in a novel blinded discovery real-time fMRI protocol 


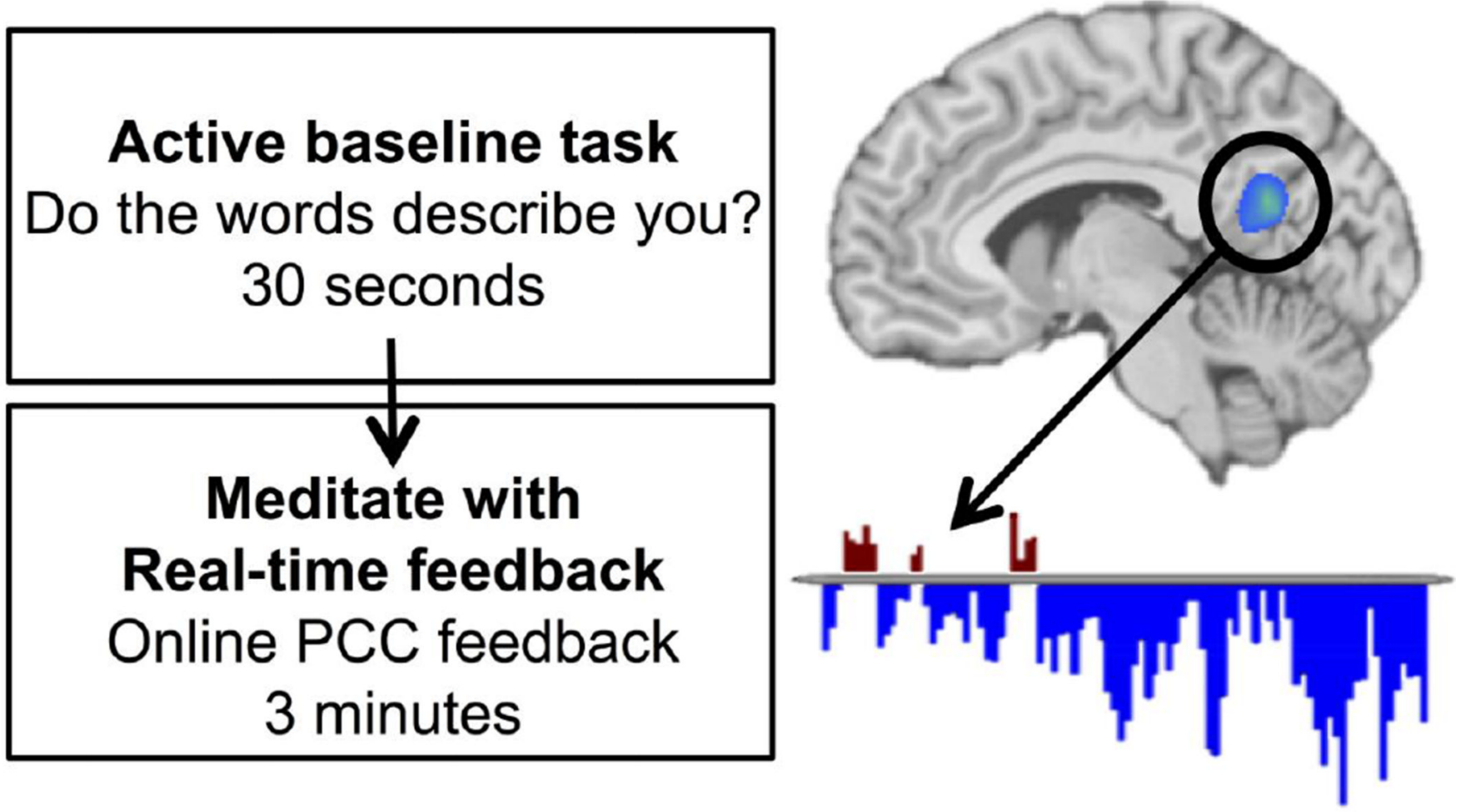

Figure 1. Real-time fMRI neurofeedback protocol for Experiment 1A

Participants performed 3 runs of a 30-second active baseline task, during which they viewed words and decided if the words described them, and a 3-minute focused attention task, during which they focused attention on the breath while viewing a real-time feedback graph representing activity in the posterior cingulate cortex (PCC). 


\section{Step 1}

Meditate on breath

Eyes open/ blank screen

Offline PCC feedback

4 runs

Step 2
Meditate on graph
Mock PCC feedback
Offline PCC feedback

3 runs

Step 3
Meditate with
Real-time feedback
Online PCC feedback

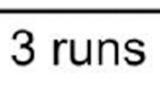

Figure 2. Real-time fMRI neurofeedback protocol for Experiment 2

Participants performed 13 runs of the same 30-second active baseline task, during which they viewed words and decided if the words described them, and a 60-second focused attention task, for which the instructions changed every 3-4 runs. For specific instructions, see Methods, Experiment 2, Blinded discovery fMRI protocol. 

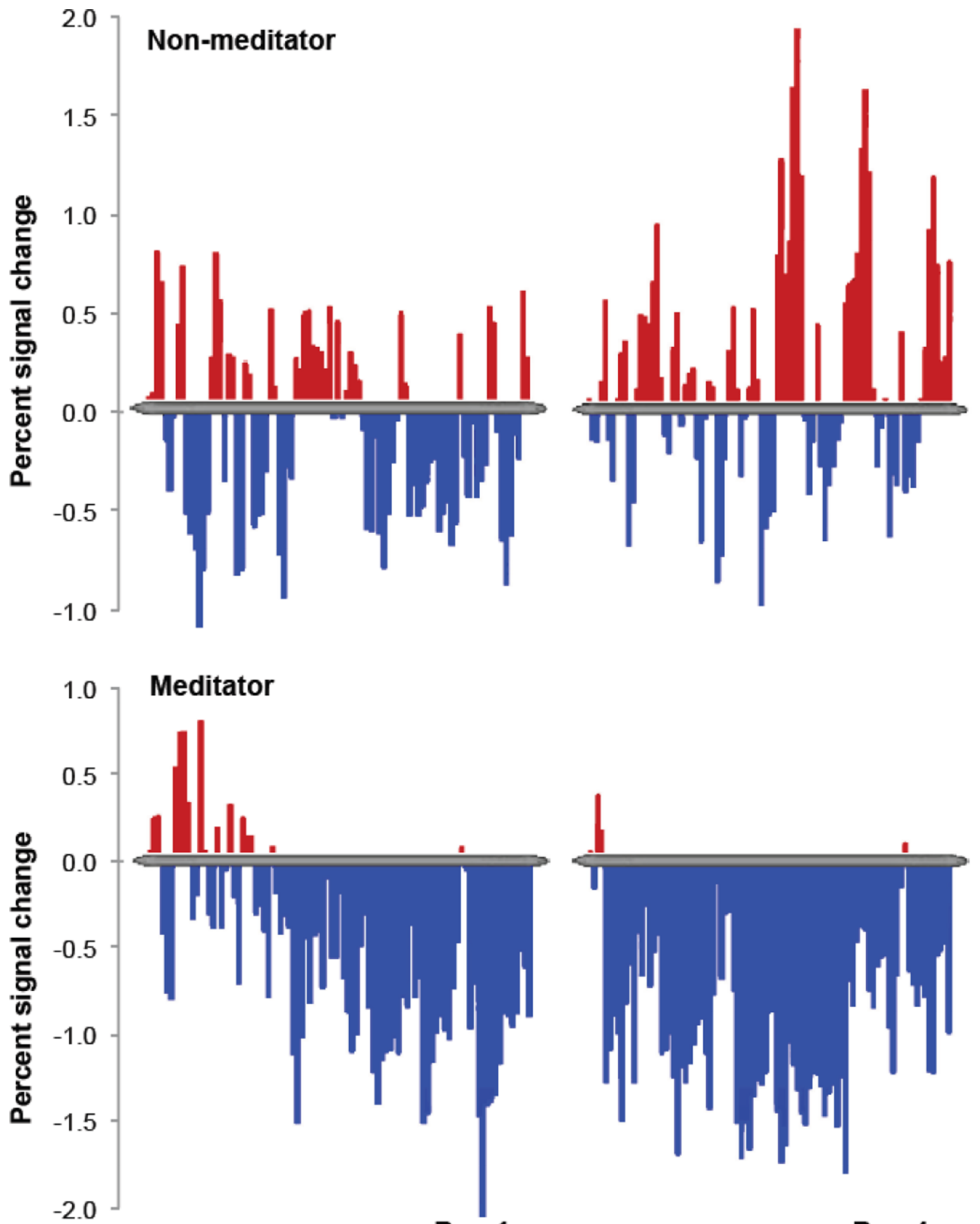

Run 1

Run 4

Figure 3. Real-time feedback from the PCC during focused attention

Mean percent signal change from the PCC during focused attention with real-time feedback is shown from an example non-meditator (top) and meditator (bottom) for run 1 and run 4, from Experiment $1 \mathrm{~A}$. 


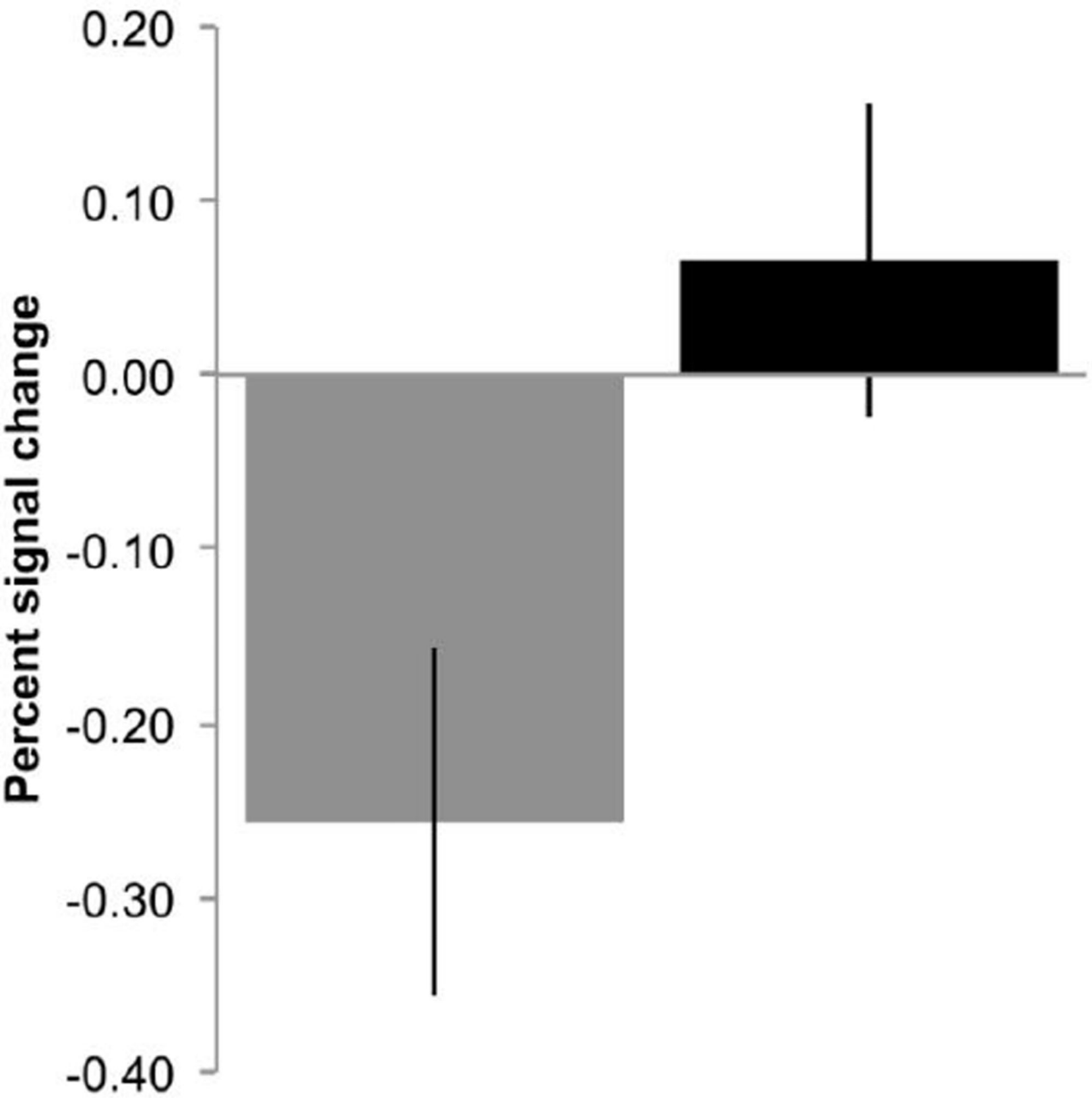

\section{Meditators}

Non-meditators

Figure 4. Experienced meditators show significantly greater deactivation of the PCC during volitional decrease of the feedback graph than non-meditators

Mean percent signal change \pm SEM in the PCC during volitional decrease of the feedback graph for meditators (gray) and non-meditators (black), from Experiment 1B, $p=.028$. 


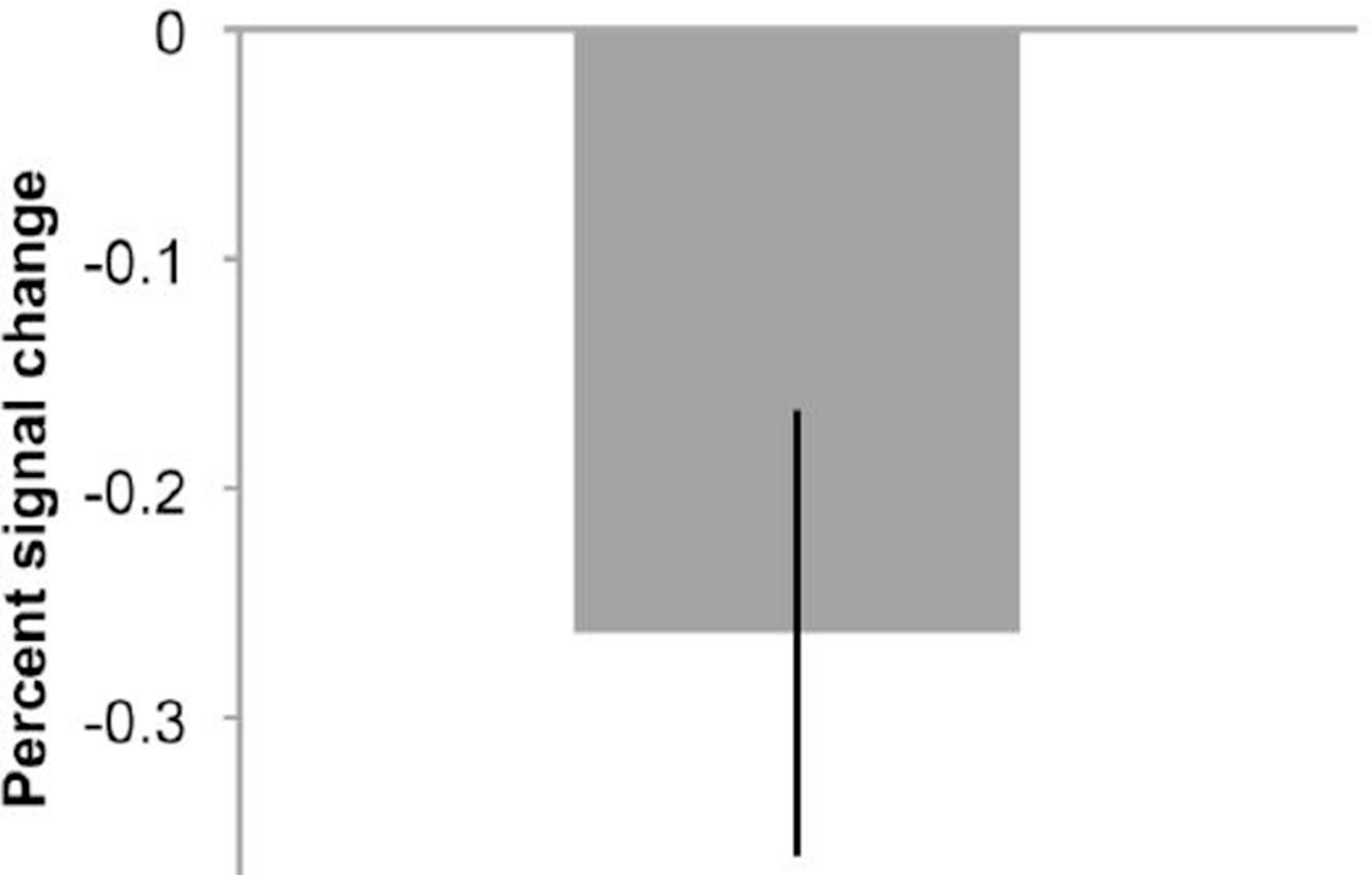

\section{Meditators}

Figure 5. Experienced meditators show significant deactivation of the PCC during volitional decrease of the feedback graph in blinded discovery protocol

Mean percent signal change \pm SEM from the PCC during volitional decrease of the feedback graph for meditators (gray), from step 4 of Experiment 2, p=.026. 


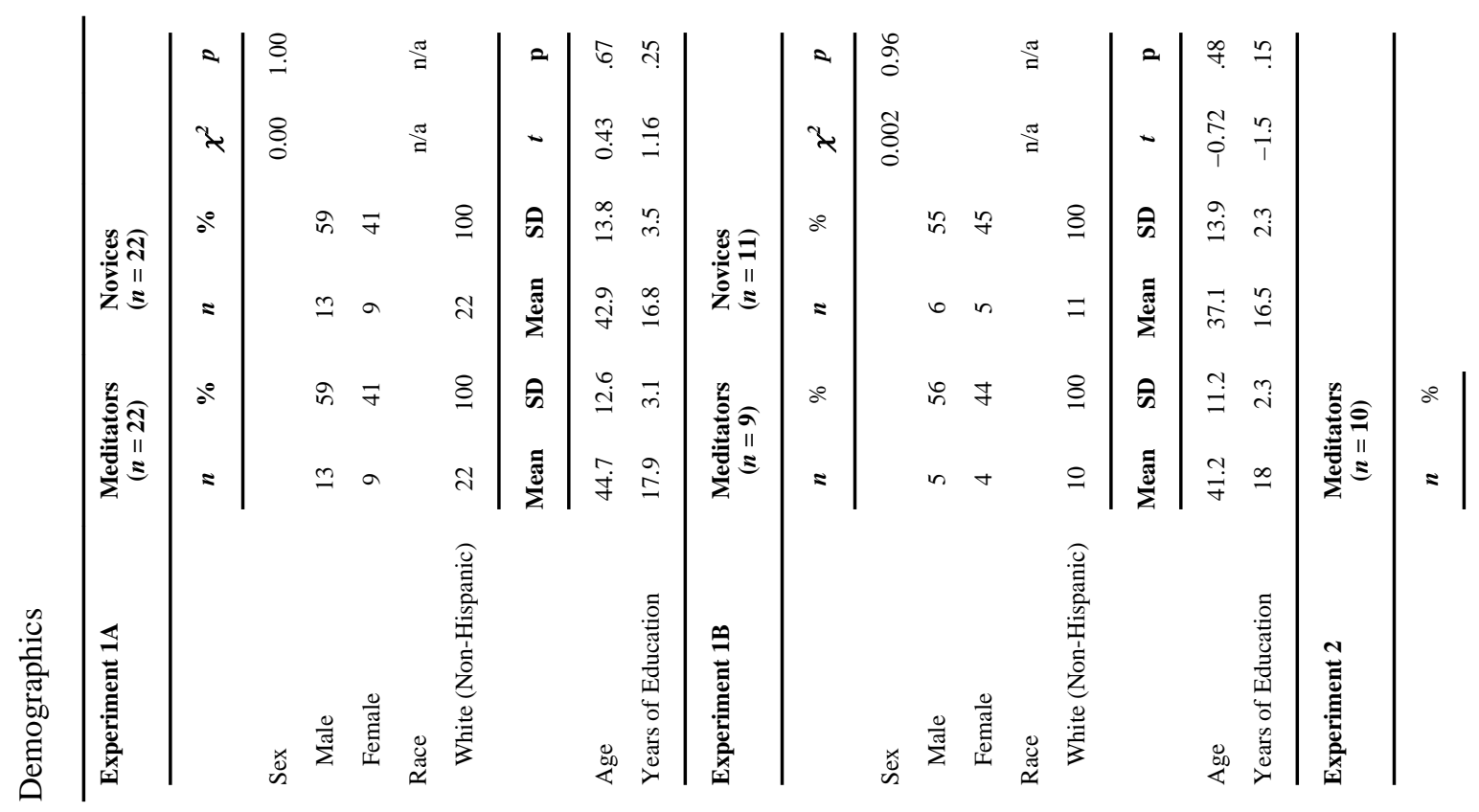




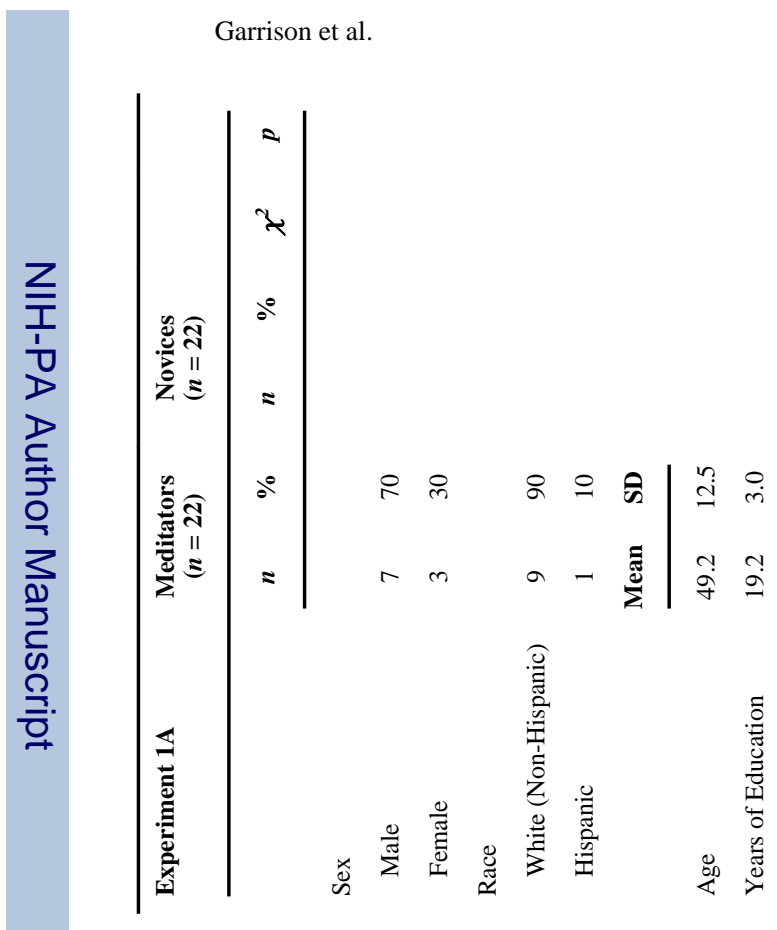

Page 21 